\title{
Emission profile variability in hot star winds
}

\section{A pseudo-3D method based on radiation hydrodynamics simulations}

\author{
L. Dessart ${ }^{1}$ and S. P. Owocki ${ }^{2}$ \\ 1 N\&S Sterrenkunde Universiteit Utrecht, Princetonplein 5, 3584 CC Utrecht, The Netherlands \\ 2 Bartol Research Institute of the University of Delaware, Newark, DE 19716, USA \\ e-mail: owocki@bartol.udel.edu
}

Received 9 November 2001 / Accepted 18 December 2001

\begin{abstract}
We present theoretical calculations of emission line profile variability based on hot star wind structure calculated numerically using radiation hydrodynamics simulations. A principal goal is to examine how well shorttime-scale variations observed in wind emission lines can be modelled by wind structure arising from small-scale instabilities intrinsic to the line-driving of these winds. The simulations here use a new implementation of the Smooth Source Function formalism for line-driving within a one-dimensional (1D) operation of the standard hydrodynamics code ZEUS-2D. As in previous wind instability simulations, the restriction to 1D is necessitated by the computational costs of non-local integrations needed for the line-driving force; but we find that naive application of such simulations within an explicit assumption of spherically symmetric structure leads to an unobserved strong concentration of profile variability toward the line wings. We thus introduce a new "patch method" for mimicking a full 3D wind structure by collecting random sequences of 1D simulations to represent the structure evolution along radial rays that extend over a selectable patch-size of solid angle. We provide illustrative results for a selection of patch sizes applied to a simulation with standard assumptions that govern the details of instability-generated wind structure, and show in particular that a typical model with a patch size of about 3 deg can qualitatively reproduce the fundamental properties of observed profile variations. We conclude with a discussion of prospects for extending the simulation method to optically thick winds of Wolf-Rayet (WR) stars, and for thereby applying our "patch method" to dynamical modelling of the extensive variability observed in wind emission lines from these WR stars.
\end{abstract}

Key words. line: formation - radiative transfer - stars: atmospheres - stars: early type - stars: mass loss

\section{Introduction}

Supersonic winds are a very fundamental feature of hot and luminous stars. The presence of such dense and fast outflows makes the spectra of such objects unequivocal, due to the numerous and strong emission lines in the optical range, as well as the distinct $\mathrm{P}-\mathrm{Cygni}$ type of profile encountered in the ultraviolet - a signature of mass outflow in interaction with a fixed light source. Although most of the energy output from these stars occurs in the far ultraviolet, these objects were for a long time only known through their optical spectra, showing a wealth of broad lines from the dominant ionised species present in the line forming regions. These are mostly from hydrogen and helium for main sequence massive OB stars while the evolved WR stars show lines from helium and nitrogen (the WN type) or helium, carbon and oxygen (the WC and WO types).

Send offprint requests to: L. Dessart, e-mail: 1.dessart@phys.uu.nl
Indeed, $\mathrm{OB}$ and $\mathrm{WR}$ stars possess fast $\left(v_{\infty} \sim\right.$ $\left.2000 \mathrm{~km} \mathrm{~s}^{-1}\right)$ and dense outflows $\left(\dot{M} \sim 10^{-5} M_{\odot} \mathrm{yr}^{-1}\right)$, which result from the very efficient momentum transfer of continuum photons to ions via line scattering and absorption. Since the first work of Lucy \& Solomon (1970), Castor et al. (1975, CAK) and Abbott (1980, 1982), a successful theory of OB star winds has been developed (Pauldrach et al. 1986), providing reliable determinations for stellar mass loss rates within the single scattering limit. However, the denser WR outflows seem to require the occurrence of multiple line scattering (Abbott \& Lucy 1985; Springmann 1994) in order to extract more efficiently the momentum contained in the radiation field and drive more mass out of the potential well of the star. This can happen only when the frequency density of opaque lines across the spectrum is high, forcing trapped photons to impart many times their radial momentum before escape (Friend \& Castor 1983; Gayley \& Owocki 1995). Unfortunately, no study so far has been able to model selfconsistently the multi-line driving of WR winds. While 
model atmospheres for WR outflows exist (Hillier et al. 1993; Hillier \& Miller 1998; Koesterke \& Hamann 1995), their steady-state, spherically symmetric approach has to assume a mass-loss rate and a prescribed velocity law.

Although such steady, smooth, spherically symmetric models are generally quite successful in describing overall wind properties, there are numerous indications that hot star winds are in fact quite structured and variable on a range of spatial and temporal scales. Largescale structure is inferred directly by explicit variability in even relatively low signal-to-noise $(S / N \sim 20)$ IUE spectra, which often show recurring, migrating "Discrete Absorption Components" (DACs) in unsaturated P-Cygni line profiles, most likely arising from co-rotating interaction regions induced in the wind outflow by surface structures or pulsations on the underlying rotating stellar surface (Owocki et al. 1995; Cranmer \& Owocki 1996). Smaller-scale, stochastic structure is inferred more indirectly from the black troughs of saturated P-Cygni line profiles, which are understood to arise from the net backscattering of multiple line resonances occurring in a highly non-monotonic velocity field (Lucy 1982, 1984). Embedded wind shocks arising from such velocity variations are moreover thought to give rise to the soft X-ray emission often observed from such hot stars (Chlebowski 1989; Lucy \& White 1980; Feldmeier et al. 1997).

But the most direct diagnostic for such small-scale structure is the explicit, small-amplitude Line Profile Variability (LPV) often detected in much higher signalto-noise ( $\mathrm{S} / \mathrm{N} \sim 100-1000)$, optical spectra obtained by ground-based monitoring of recombination emission lines in both OB (Eversberg et al. 1998 on $\zeta$ Puppis) and WR stars (Robert 1992; Lépine \& Moffat 1999). Wavelet analyses (Lépine 1995; Lépine et al. 1996) have yielded quite extensive observational constraints on the statistical properties of the LPV. For example, the observed systematic shift of LPV patterns from line centre to wing provides a dynamical diagnostic of the apparent outward acceleration of the associated wind structures. But thus far, analyses have centred on phenomenological descriptions like the Discrete Wind Emission Elements (DWEEs; Lépine \& Moffat 1999), which are not directly linked to physical properties. As such, the fundamental physical and dynamical properties of wind structure that gives rise to the observed LPV are still largely unknown.

The long-term objective of the analyses initiated in this paper is to develop such physical and dynamical understanding of the small-scale wind structure associated with such LPV. The general approach is to examine the degree to which structure arising from the intrinsic dynamical instability of line-driving of such winds (Owocki \& Rybicki 1984, 1985) could yield LPV with the observed properties. However, numerical simulation on the nonlinear evolution of such instability-generated structure requires a computationally expensive non-local integration of the line-force, for example using the Smooth Source Function (SSF) formalism (Owocki 1991; Owocki \& Puls 1996, 1999). As such, simulations carried out thus far have generally been limited to just one-dimension (1D), modelling the time-dependent, radial evolution of wind structure. Owocki (1999) used an approximate 3-ray method in 2D hydrodynamics simulations that provided some initial results on the lateral coherence of instability generated structures, and how these are affected by stellar rotation. More general, extendable approaches for modelling $2 \mathrm{D}$ or $3 \mathrm{D}$ instability generated structures might become computationally feasible with new, massively parallel computer clusters, but the detailed methods and codes still await development.

In this paper, we derive emission line profiles from such models of instability-generated structure, applying a new implementation (developed by LD) of the SSF lineforce method within a $1 \mathrm{D}$ operation of the standard hydrodynamics simulation code ZEUS-2D, as described in Appendix A. Under the simplifying approximation of optically thin emission, we first compute the LPV under the literal assumption that 1D radial structures extend over spherically symmetric shells (Sect. 2), and find thereby that the resulting LPV are quite unrealistic as compared to observations. We thus introduce (Sect. 3) a new "patch method" for mimicking a more realistic 3D wind structure by using randomised time intervals of $1 \mathrm{D}$ simulation models to represent wind structure along radial rays that extend over a selectable patch-size of solid angle. We then illustrate how the choice of a suitable small patch-size can lead to a much more realistic LPV. Finally, we conclude in Sect. 4 with a discussion of our results, highlighting some important physical issues for future investigation.

\section{Synthesising emission line profiles from a structured wind: The shell case}

Before discussing the details of the patch emission approach, let us summarise our basic method for computing the line profile emission for a spherically symmetric outflow, both with and without extensive small-scale, radial structure.

\subsection{Line emission for a spherically symmetric wind}

Following Owocki \& Cohen (2001), we write the expression for the line emission at the observer's wavelength $\lambda$, from a wind element with the spherical coordinates $(r, \theta, \phi)$, with $\mu \equiv \cos \theta$

$L_{\lambda}=4 \pi \int_{-1}^{1} \mathrm{~d} \mu \int_{0}^{2 \pi} \mathrm{d} \phi \int_{R_{\star}}^{r_{\max }} \mathrm{d} r r^{2} \eta_{\lambda}(r, \mu, \phi)$.

The wind volume is limited by spheres at $R_{\star}$ and $r_{\max }$, but can be built with an arbitrary structure. In this initial paper, we assume a simple optically thin line emission, deferring to future work inclusion of the effects of continuum or line absorption and/or scattering within the wind (Owocki \& Cohen 2001; Ignace \& Gayley 2001). Thus, the wind emissivity at $(r, \mu, \phi)$ and at wavelength $\lambda$ is

$\eta_{\lambda}(r, \mu, \phi)=c s t \times \rho^{2}(r) f(r) \delta\left(\lambda-\lambda_{0}\left(1-\frac{\mu v(r)}{c}\right)\right)$ 
where $f(r)$ accounts for the radial dependence of the emission, e.g. due to ionization and excitation. In this work, we generally take this to be unity between specified lower and upper radii $r_{1}$ and $r_{\mathrm{u}}$, and zero otherwise. Since we are interested only in relative profiles changes, the overall multiplicative factor $c$ st is also set to unity.

Normalising the wavelength by $x=\left(\lambda / \lambda_{0}-1\right) c / v_{\infty}$, and using the property $L_{\lambda} \mathrm{d} \lambda=L_{x} \mathrm{~d} x$, we have

$L_{x} \propto \int_{-1}^{1} \mathrm{~d} \mu \int_{0}^{2 \pi} \mathrm{d} \phi \int_{R_{\star}}^{r_{\max }} \mathrm{d} r r^{2} \rho^{2}(r) f(r) \delta\left(x+\frac{\mu v(r)}{v_{\infty}}\right)$.

Here we have scaled the wavelength in units of the CAK terminal speed $v_{\infty}$, so that in an unperturbed CAK flow, the line profile is bounded between the normalised wavelengths -1 and 1 . However note that, in a structured wind, some of the material can have speeds $v>v_{\infty}$, and so lead to profile variations beyond the range $-1<x<1$.

If we consider the emission from a narrow radial shell of width $\mathrm{d} r$ at radius $r$, then integration over $\mu$ gives

$\mathrm{d} L_{x}(r) \propto \begin{cases}2 \pi\left(v_{\infty} / v(r)\right) r^{2} \rho^{2}(r) \mathrm{d} r & \text { for }|x| \leq v(r) / v_{\infty} \\ 0 . & \text { otherwise },\end{cases}$

thus showing that the line emission from this thin shell forms a flat-topped box profile over the wavelengths $x$ within $\pm v(r) / v_{\infty}$. We build the total profile by integrating over all shells,

$L_{x}=\int_{R_{\star}}^{r_{\max }} \mathrm{d} r\left[\frac{\mathrm{d} L_{x}(r)}{\mathrm{d} r}\right]$,

applying the wavelength test to determine over what fraction of the profile the contributions are made. The profile from this optically thin approach is therefore built up from flat emission intervals that start out being quite narrow near the wind base and become wider further out. If the onset of wind emission is set to a point where the wind has reached terminal velocity (i.e. $v\left(r_{1}\right) \approx v_{\infty}$ ), these slabs all have the same width and the computed profile possesses a square shape, in agreement with observations (a beautiful example is the Ne[III] $15.55 \mu \mathrm{m}$ line observed in the ISOSWS spectrum of WR 11, alias $\gamma$ Velorum, see Dessart 1999).

\subsection{Application to spherical wind snapshots}

It can be fruitful to study a few examples in order to get more accustomed to the scientific core of our presentation. By considering the simple case of full wind symmetry about star centre, we can understand better which part of the wind contributes to which wavelength range.

For this example, we allow nearly the entire wind to contribute to the emission, i.e. $r_{\mathrm{l}}$ and $r_{\mathrm{u}}$ defined above correspond respectively to $1.5 R_{\star}$ and $r_{\max }=10 R_{\star}$. We exclude the innermost wind region because, even though the volume of integration is small, the very high density makes the profile dominated by the small velocity region, something that is not observed in emission lines anyway, but would also make the analysis of the computed profile difficult. As we shall discuss further in a follow-up paper, the emission from these high density regions suffers a very strong attenuation due to continuum absorption.

In Fig. 1 we illustrate the emission line formation in two spherical, isotropic models, comparing the results for a steady, smooth, CAK wind (left) with those at a fixed time snapshot of a variable, structured outflow (right) computed with the SSF formalism described in Appendix A. The bottom panels show the radial variation of velocity and density at a fixed time snapshot. The next panels above these show the computed line profiles for each model. The top panels break down the profile into the radial contribution of emission vs. wavelength.

At any wavelength $x$ measured from line centre, or its corresponding Doppler shifted velocity $v$, the emission contribution comes from points with higher intrinsic velocities (and same sign). In a smooth wind, this concerns points lying along $\mu=1$ and with a velocity greater than $v$, while as we move to larger line of sight angles, only regions of greater and greater intrinsic velocity contribute (this is merely to put in words the implication of the geometry of isovelocity curves in an expanding flow). However, in a structured wind, the velocity is non-monotonic, so that for a fixed radius, one can in principle find regions of higher and smaller heights that can contribute at the profile frequency $x$, over a restricted set of values of $\mu$. If the wind is highly structured, this can amount to hundreds of such regions, which are said to be in resonance. In that case, there is no simple link between a given profile wavelength $x$ and a wind radius $r$ : the profile construction is done via a very non-local assembly of individual contributions, making the analysis of the global profile non-trivial.

In the top panel of Fig. 1, the computed contribution from a region $\Delta r$ to the wavelength band $\Delta x$ is displayed in an arbitrary unit and in a logarithmic scale, and shows the relative emissivity of wind regions at selected profile velocity bins. In a smooth wind (left panel), the wind emissivity gradually diminishes with height, in a very continuous manner, whereas in the structured case, the maximum velocity for the contribution keeps changing with radius. Some regions even hardly contribute at all, e.g. the low density regions at $2.5,4.7,6$. and $7.3 R_{\star}$. Note also the spiky nature of the dense shells, which appear as well in the top plot as bright strips across the wavelength range, but despite their presence, the global emission profile shows no spikiness whatsoever but rather a series of small but discrete steps. This fact severely hinders an unique derivation of wind structure properties, since many different wind structures can produce a given, spatially integrated emission line profile.

Moreover, the top of the profile limited by $\pm v_{\text {top }}$ (which spans the line of sight velocity bounds of the onset of the emission region) is a sum of the contributions of all regions having a line of sight velocity greater than $\pm v_{\text {top }}$. The flat portion of the profile is then at every instant a sum of all the contributions from all wind points moving faster than $v_{\text {top }}$. Their temporal average contribution 

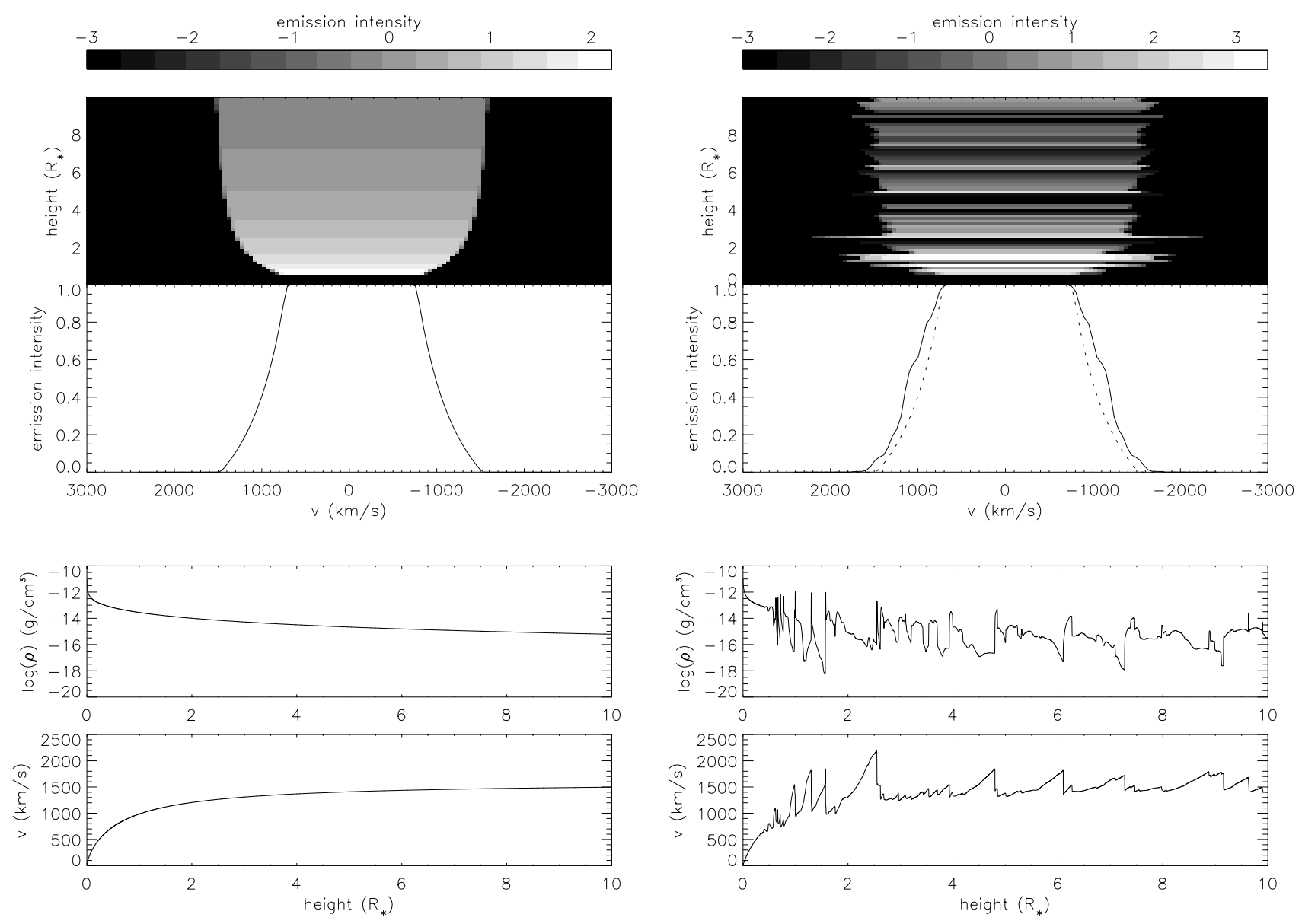

Fig. 1. Calculation of two emission line profiles assuming wind isotropy, in the smooth case on the left and the structured case on the right. From bottom to top, we display the velocity and density as a function of height above photosphere, then the computed line profile in velocity space, centred on its rest wavelength. The top image shows the relative contributions to the profile (logarithmic scale), as a function of wavelength and height above photosphere for the entire wind emitting volume.

is the same at all wavelengths bounded by $\pm v_{\text {top }}$ hence no variation from wavelength to wavelength can be witnessed in the range limited by $\pm v_{\text {top. }}$. So, whether one uses a structured or a smooth wind input to the profile calculation, the assumption of spherical symmetry implies the flux constancy in the top part of the profile.

Finally, it is nice to observe that the shape of the contributing region in the wavelength-radius space is just a reflection of the variation of the velocity with radius, a fact which is not so surprising after all, since the emission is really bounded by radial as well as velocity limits.

\subsection{Synthetic profile monitoring assuming a spherically symmetric wind}

Now, we wish to assess the emission LPV this simple minded model would predict, to check whether the assumption of spherical symmetry, which is for example assumed to be adequate in all model atmosphere calculations for hot stars, does a good job at reproducing any of the main observed characteristics shown in Lépine \& Moffat (1999) for example.
We have for that purpose calculated the emission line profile arising from a spherically symmetric wind using a large database of one dimensional wind snapshots. These outputs are recorded as a time sequence evolution of the wind structure calculated by ZEUS-2D, and so we computed a series of profiles following the method described in the beginning of this section, each being calculated using the $1 \mathrm{D}$ structure at $t$, with $t$ running over about ten hours, with one output every $6 \mathrm{~min}$. We show the result of this simulation in Fig. 2, by displaying the deviation from the mean profile as function of time and frequency, as well as providing the overall mean profile and residual. The striking characteristics of this simulation is that the migrating sub-peaks so characteristic of emission LPV are only present in the wings of the line, while the flat top profile remains flat stricto sensu.

Compared with observations of LPV, the prediction of a variability confined to the line wings is problematic. Indeed, Lépine \& Moffat (1999), amongst many other sources for such observations, discuss the presence of migrating features at the top of emission line profiles, while the line wings are the site of no significant variability, possibly due to the sharp decrease in wind emissivity at 

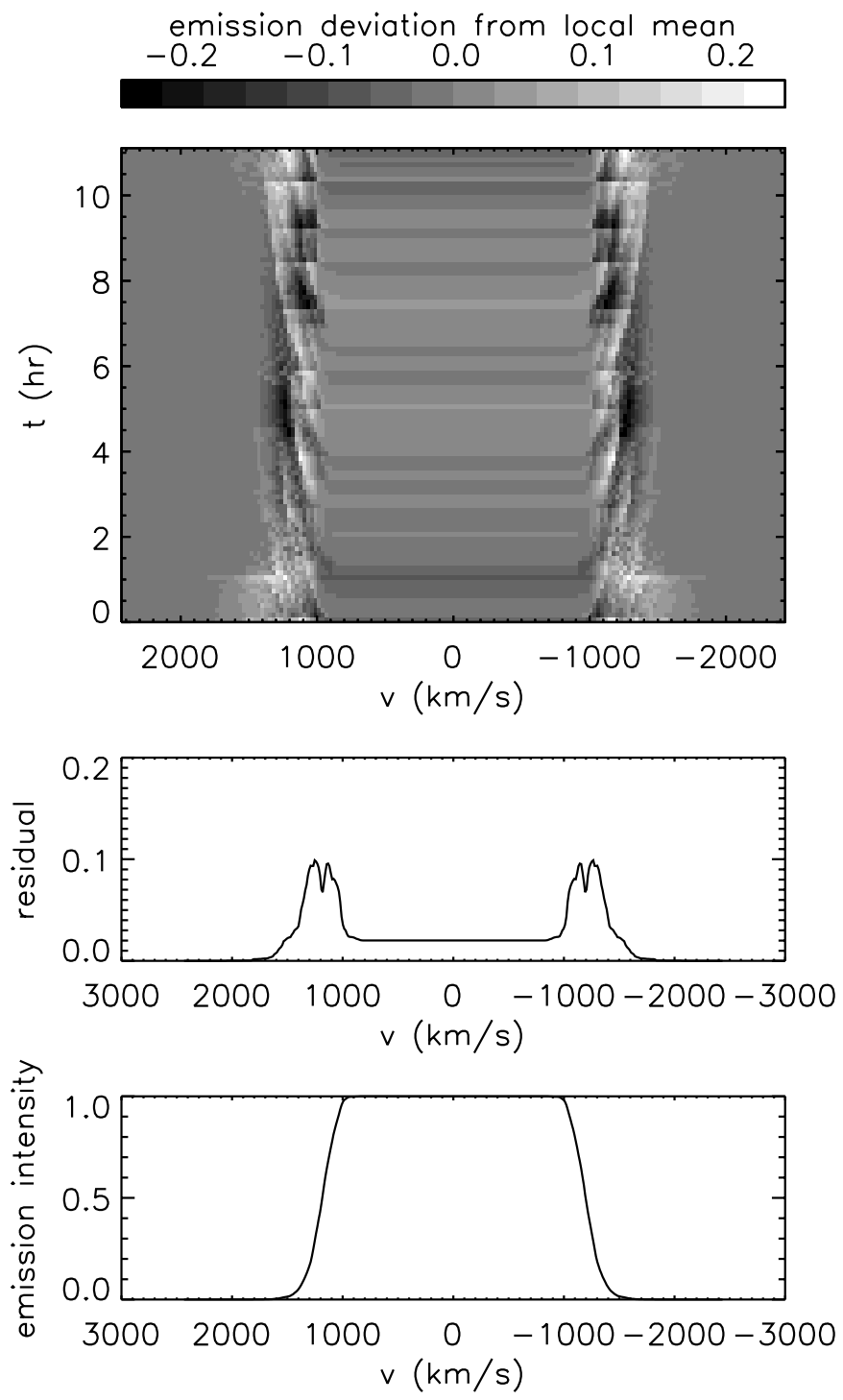

Fig. 2. (Top) Gray-scale image of the temporal evolution of the profile deviation from the local mean over the period comparable to a long night of observations. The wind is assumed to be structured into spherically symmetric shells. Note how the velocity to velocity variability at $t$ is confined to the line wings, as revealed by the (middle) plot of residuals. The only variability observed in the flat top region corresponds to an overall fluctuation of the global emissivity in the line, which scales sensitively with the number of dense shells in the emission region (the larger the extent of the line emission region, the smaller this fluctuation). The (bottom) plot displays the average of the sequence of computed profiles.

those line of sight velocities. To recover the wavelength and temporal dependence of the wind emissivity, we relax the assumption of global spherical symmetry and design the "patch method" described in detail in the next section.

\section{Synthesising emission line profiles from a structured wind: The "patch method"}

Let us now consider an approach to generalise the spherically symmetric emission calculation from the previous section to a more realistic $3 \mathrm{D}$ structure. As already noted in the introduction, the non-local nature of the line-force computation has thus far severely limited attempts to extend direct simulations of this instability beyond just $1 \mathrm{D}$, radial models. In principle, effects from rotation, gas pressure, and even lateral components of the line-force (Rybicki et al. 1990; Gayley \& Owocki 2000; Owocki et al. 1996; Cranmer \& Owocki 1996) could all play some dynamical role in the lateral coherence and propagation of flow structure. Nonetheless, given the general dominance of the radial component of the radiative driving and acceleration in accelerating the overall radial outflow, it seems a reasonable first approximation to model the dynamics within the context of $1 \mathrm{D}$ radial flow streams, deferring to future work any treatment of such terms for lateral dynamics and transport.

This warrants the mapping of the wind volume with a number of independent cones, or patches, of characteristic angular diameter $\alpha$, as viewed from the star centre. The first task is then to set up the wind geometry. Let us consider an observer viewing the star along a coordinate direction $z$. We identify the $(x, y)$ plane to be perpendicular to this ray and containing the star centre. Using the usual spherical coordinates, $\theta$ is the angle between the $z$ axis and the radial direction at some radial coordinate $r$, while $\phi$ is the azimuthal angle that an $(x, y)$ projection of the radial ray makes with the $x$-axis. The full wind volume is covered with $r \in\left[R_{\star}, r_{\max }\right], \theta \in[0, \pi]$, and $\phi \in[0,2 \pi]$.

For a chosen patch angular extent $\alpha$, we first assign one single patch each to the degenerate directions $\theta=0$ and $\theta=\pi$. For the remaining solid angle in the range $\theta \in[\alpha / 2, \pi-\alpha / 2]$, we first split the $\theta$ direction into $n=(\pi / \alpha)-1$ annuli of equal $\theta$-width $\alpha$, with the $i$ th annulus centred on $\theta_{i}=i * \alpha, 1<i<n$, and having a circumference $l_{i}=2 \pi \sin \left(\theta_{i}\right)$. We then further divide each annulus into patches of roughly equal characteristic $\phi$-width $\alpha$, with the number of patches in the $i$ th annulus given by $m_{i}=m\left(\theta_{i}\right)=\operatorname{Int}\left[l_{i} / \alpha\right]=\operatorname{Int}[2 \pi \sin (i \alpha) / \alpha]$, where Int $[x]$ gives the nearest integer to $x$. The total number of patches over the sphere thus scales as

$$
N=2+\sum_{i=1, n} m_{i} \approx \frac{2 \pi}{\alpha} \sum_{i=1, n} \sin \theta_{i} \approx \frac{2 \pi}{\alpha^{2}} \int_{0}^{\pi} \mathrm{d} \theta \sin \theta=\frac{4 \pi}{\alpha^{2}}
$$

where the latter expressions apply to the case of small patch size, $\alpha \ll 1$, for which the integer sum can be approximated as a continuous integral over $\theta$.

Note that the patches are not round but rather are roughly square-shaped sections of a sphere, with an extent $\alpha$ in both the $\theta$ and $\phi$ directions. This leads to some minor ambiguities near corners, but the contribution of these locations is a small fraction of that from the total patch.

As a specific example, if we choose $\alpha=3 \mathrm{deg}=\pi / 60$, then the $\theta$ direction is split into $n=(180 / 3)-1=59$ annuli (in addition to the two singular patches at $\theta=0$ and $\pi$ ). The number of patches in each annulus is $m_{1}=6$ at $\theta_{1}=\alpha, m_{2}=12$ at $\theta=2 \alpha, \ldots, m_{30}=120$ at $\theta_{30}=$ $30 \alpha=\pi / 2$. Direct evaluation of the sum gives a total 
number of patches $N=4586$, which is quite close to the approximate value, $N \approx 4584$, given by the above scaling expression for $N$.

A central feature of this technique is that it applies for any detailed physical properties of the individual patches. For two patches within a given annulus, the line of sight angle is the same. If moreover they have the same density and velocity properties they will contribute the same amount and at the same wavelengths in the profile. Compared to the total contribution of the annulus, a single patch contributes in proportion to its $\phi$ extent in the annulus. Since all patches are identical, this is $2 \pi / m_{i}$. It is clear that when we run over the $m_{i}$ patches in the annulus, we get a $2 \pi$ weight, which is the weight of an annulus in the shell approach. So this way, we know that our geometries are compatible.

To calculate the emergent profile from a $3 \mathrm{D}$ wind made of such patches, we sum up the contributions from all patches, much as done above for spherical shells. For each of the $m_{i}$ patches in each annulus $\theta_{i}$ at each radius $r$, the differential contribution to the emission is given by

$\mathrm{d} L_{x}\left(r, \theta_{i}\right) \propto \begin{cases}\frac{2 \pi}{m_{i}} \frac{v_{\infty}}{v(r)} r^{2} \rho^{2}(r) \mathrm{d} r & \text { for } x_{\min , i}<x<x_{\max , i} \\ 0 . & \text { otherwise }\end{cases}$

where $x_{\min , i}=\cos \left(\theta_{i}+\alpha / 2\right) v(r) / v_{\infty}$ and $x_{\max , i}=\cos \left(\theta_{i}-\right.$ $\alpha / 2) v(r) / v_{\infty}$. For small patches $\alpha \ll 1$, the wavelength range of each patch's emission scales approximately as $\Delta x_{i} \equiv x_{\max , i}-x_{\min , i} \approx \alpha \sin \theta_{i} v(r) / v_{\infty}$. For the front and back patches at $\theta=0, \pi$, this emission has the minimum wavelength range $\Delta x_{\mathrm{o}}=\alpha^{2} v(r) / 2 v_{\infty}$.

In practice we compute the emergent line profile over a fixed wavelength array that typically contains several hundred elements spaced in equal intervals $\Delta x$ to span the full range $\pm v_{\max } / v_{\infty}$, where $v_{\max }$ is the maximum velocity in a given structured wind model. For typical patch sizes of order $\alpha \sim \pi / 60 \sim 0.05$, we see that those patches with substantial velocity transverse to the line of sight will emit in a wavelength range $\Delta x_{i} \sim 0.05$ that can span several wavelength grid points with separation $\Delta x \sim 0.01$. However, patches in the slow-moving inner wind, or directed nearly at $\theta=0, \pi$ - which thus have small transverse velocities - can have a quite narrow emission range, e.g. $\Delta x \sim \alpha^{2} \sim 0.002$, which thus can fall entirely between wavelength points separated by $\Delta x \sim 0.01$. In general then, the emission from many individual patches contributes to appropriate bins in the assumed wavelength grid, sometimes using linear interpolation to weight the relative proportion to the nearest neighbouring bins. With this approach, we find that a few hundred wavelength points are sufficient to represent the emergent emission profile to within an error of $<1 \%$, irrespective of the patch size $\alpha$.

We are now in a position to carry out more realistic profile simulations, based on calculations of the wind structure using our radiation hydrodynamics code described in Appendix A.
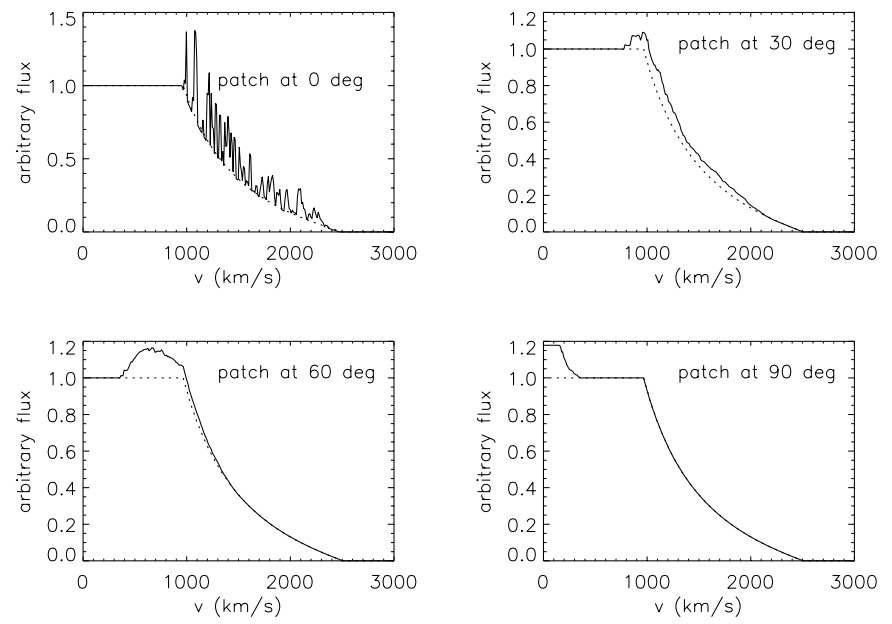

Fig. 3. We present a series of profile simulations obtained with the following assumptions. The wind is fully symmetric and smooth apart from one ray, with a sub-tended angle of $9 \mathrm{deg}$, and a line of sight angle chosen as 0 (top left), 30 (top right), 60 (bottom left) and $90 \mathrm{deg}$ (bottom right). The superimposed dotted line is the predicted profile, had the wind structure been fully smooth.

\subsection{Application to a smooth wind with a single patch of structure}

Before treating the general case of full wind asymmetry in the next section, it is instructive to consider the line emission resulting from a single solid-angle cone/patch of structured flow embedded in an otherwise smooth wind. We wish in particular to examine how the observable LPV depends on the angular size $\alpha_{\mathrm{c}}$ of the patch, and on the line of sight angle $\theta_{\mathrm{c}}$ of its centre. We first compute the overall smooth wind profile, and then subtract off the smoothwind contribution from the patch, replacing it with the patch contribution from the structured model. In fact, if one were interested in the profile subtracted from its average, then one would need only to compute the profile contribution of one ray, giving it a weight that accounts for its patch extent in the full wind. Figure 3 shows the total profile calculated for four different cases of a smooth wind with one structured patch, chosen to be at $\theta_{\mathrm{c}}$ of 0,30 , 60 and 90 deg to the line of sight, with a sufficiently large patch size $\left(\alpha_{\mathrm{c}}=9 \mathrm{deg}\right)$ to be apparent in even the diluted, full profile. Note that when the patch is at $\theta_{c}=0$, the intensity bumps are both narrow and strong, with discrete wavelengths that span the entire wavelength range accessible to their hemisphere position (red or blue part of the profile for back or front hemisphere of the observable star). For increasing $\theta_{\mathrm{c}}$, the emission excess is no longer isolated at distinct wavelengths related to individual density enhancements, but rather is bunched together. The accessible wavelength range over which they contribute becomes narrower and moves towards the line centre. Note that, within this simple case of isotropic, optically thin emission, the total emission contribution from this structured patch is independent of $\theta_{\mathrm{c}}$ : it is merely the way this contribution is spread over the profile. 
It can be worthy to mention that the frequency extent of a patch contribution is determined primarily by the angular extent of the cone if the patch lies near the mid-plane (see the discussion at the beginning of Sect. 3), and by the intrinsic velocity "extent" of the line emission region for patches nearer the direct line of sight.

\subsection{Application to a fully structured model}

Let us apply the "patch method" described above to simulate the emissivity of a wind with $3 \mathrm{D}$ structure. We make no assumptions about the radial structure of the patches but rather make a random allocation of ZEUS-2D individual snapshots with patch layouts. We proceed as follows: at $t=0$, we make this random allocation out of a sample of 400 sequential one dimensional datasets and we memorise for all patches the snapshot time used. We then calculate the emission profile arising from such a $3 \mathrm{D}$ wind and increment in time. For those profile simulations, the time increment is $400 \mathrm{~s}$, while the time increment is $100 \mathrm{~s}$ in the ZEUS-2D simulations. So, patch number $i$ which had at $t=t_{1}$ the snapshot number $n$, will have at $t=t_{1}+\Delta t$ the wind snapshot number $n+4$. Making sure that this bookkeeping is done properly, the profile sequence is done in a trivial way.

We defer to a later study a comprehensive coverage of all parameters governing the "patch method" described here. However, to propose a bridge from the full spherically symmetric case of Sect. 2 to the full anisotropic case, we describe the LPV computed for a wind volume made up of very large patches - after all, one could view the spherically symmetric case as a single patch calculation with a $360 \mathrm{deg}$ angular extent. For a patch-size of $9 \mathrm{deg}$, we show in Fig. 4 the time evolution of the profile deviation from its local mean value, together with the residual at all frequencies. This example conveys one of the core implications of our "patch method". Compared to Fig. 2, we clearly observe a non-zero residual within the profile top part, and can identify over the 10 hour simulation a number of migrating sub-peaks. Whereas in the former spherically symmetric case the variability was concentrated entirely to the profile wings, it is now present all over the profile. Unfortunately, this simulation disagrees with observations on two points: the level of profile fluctuations is a bit too large (up to 20\%), and the migrating features are concentrated at the edges of the profile. The profile width contribution of the near mid-plane patches is in that case terribly large (as discussed in Sect. 3), making the variable patterns in the flat top part very broad, while the reduced wavelength coverage of the wind clumps situated nearer the direct line of sight give rise to an observable set of narrower migrating patterns. To reduce the overall level of variability as well as the profile widths of migrating patterns, one can simply increase the number $N$ of patches in the wind - decrease the patch angular size so that the stochastic cumulative sum of local emissivities will suffer the $1 / \sqrt{N}$ more severely and the mid-plane patches will cover a more restricted frequency range.
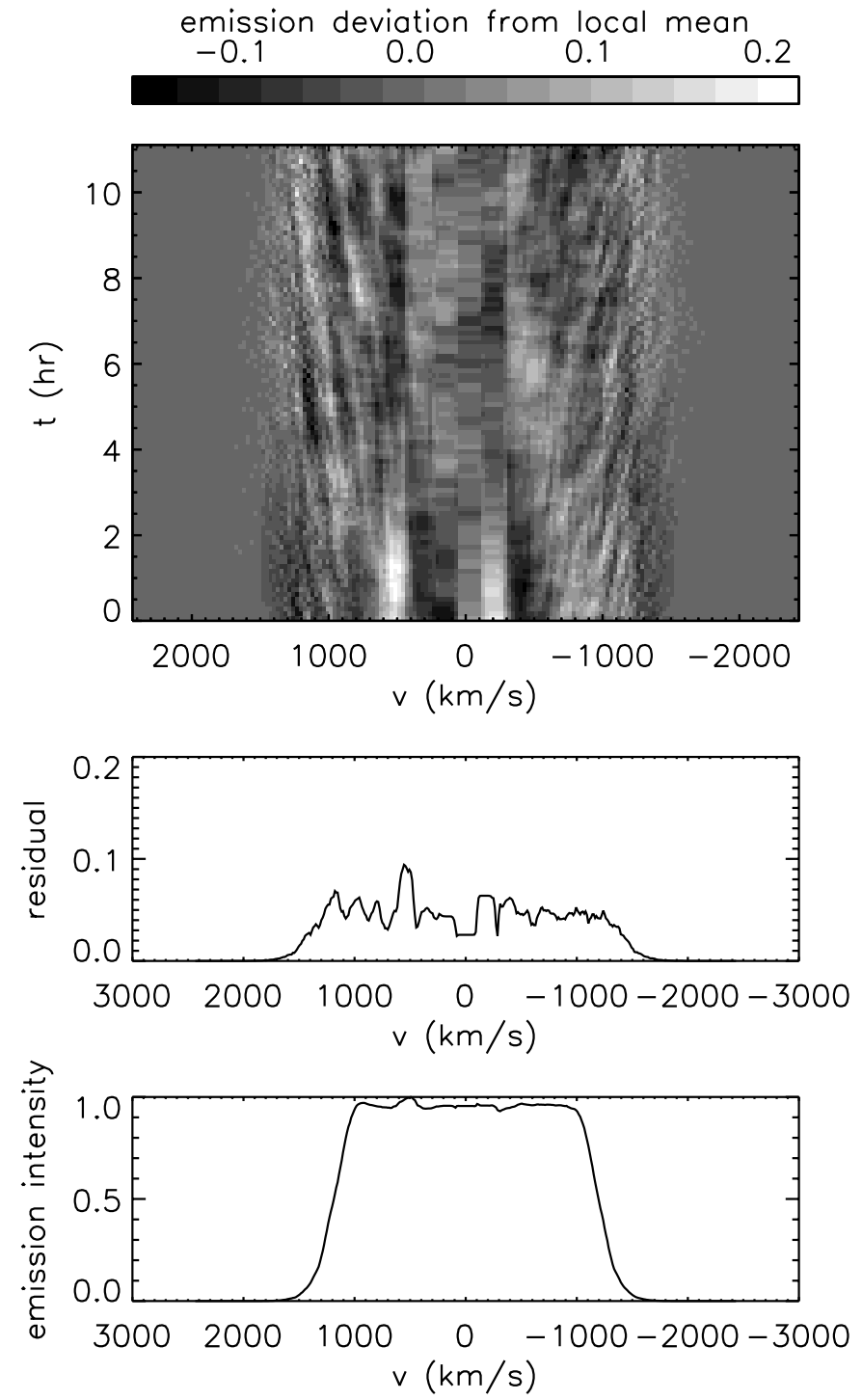

Fig. 4. Same as Fig. 2 but now using a fully asymmetric wind, composed of 506 independent cones with a sub-tended angle of 9 deg. Note the stark contrast of the calculated variability compared to Fig. 2. The profile shows a frequency to frequency non-zero residual at all profile locations, instead of a segregation to the line wings. However, the migrating sub-peaks are mostly concentrated in the line profile wings, while the level of variability itself is much larger than observed in Lépine \& Moffat (1999).

The simulation shown in Fig. 5 uses a reduced patch size of $3 \mathrm{deg}$ and provides a satisfactory match between the observed and synthetic LPV. This simulation reproduces very well the main characteristics of observed LPV outlined in Lépine \& Moffat (1999), i.e. the level of profile fluctuation is now of the order of a few percents and ubiquitous throughout the profile width, while the fundamental "migrating" behaviour of sub-peaks is well represented throughout the full profile width. We can therefore conclude that our test on the adequacy of the radiative instability generated structures to reproduce the essential features of observed LPV is successful. 

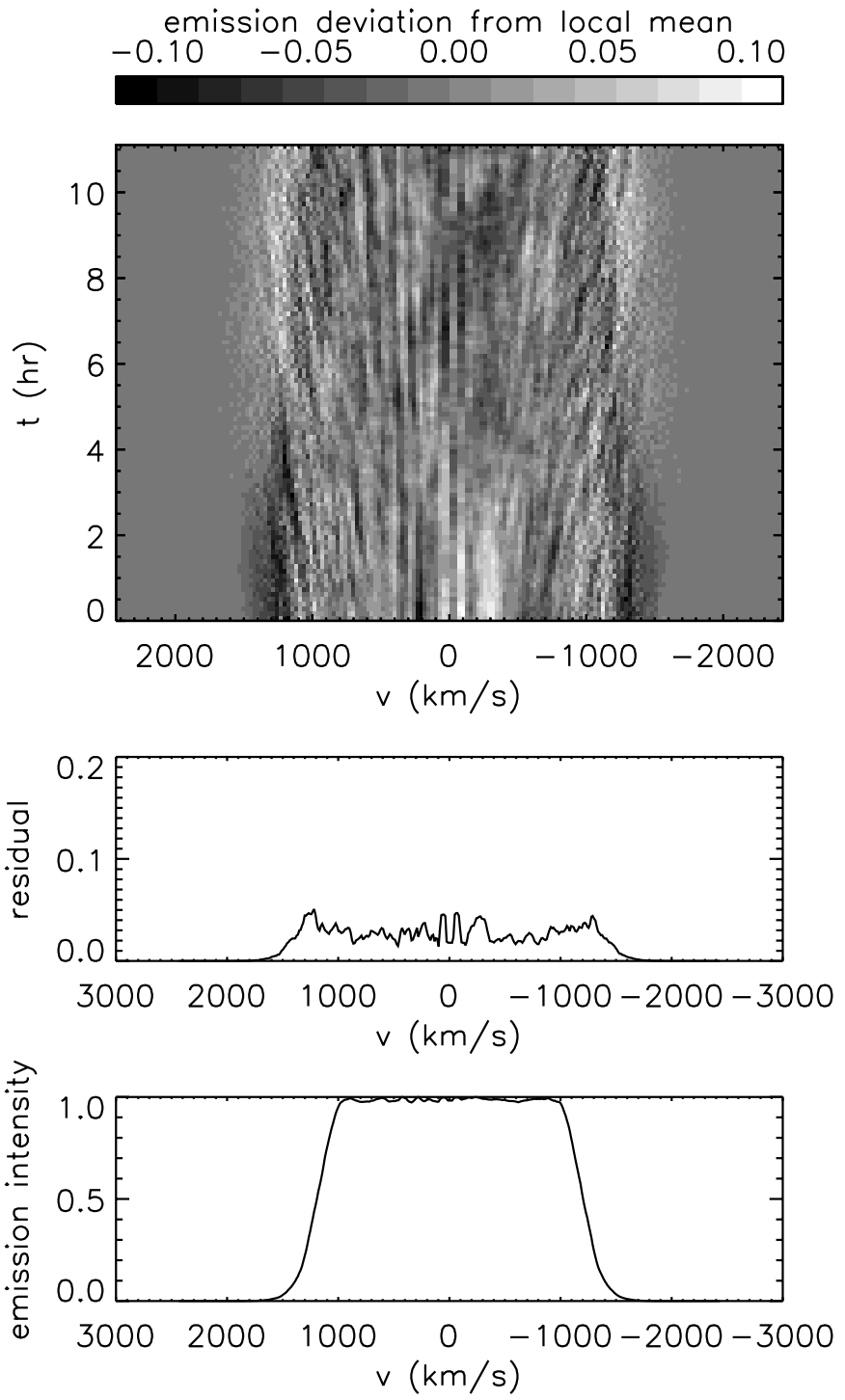

Fig. 5. Same as Fig. 2 but now using a fully asymmetric wind, composed of 4586 independent cones with a sub-tended angle of 3 deg. Note the subtle change from the previous figure. Migrating features can be found all over the profile, with a lower level of variability compared to the 9 deg patch case.

\section{Summary and conclusions}

In this paper, we have presented a new method to simulate the emission LPV, using hot star wind structure as generated by radiation hydrodynamics simulations. The novelty of this work resides in our use of physical models of the radiative instability generated wind structure to compute the wind emissivity, rather than assuming a mathematical description of the wind properties. The "patch method", described in Sect. 3 and at the core of this paper, proposes a pseudo-3D treatment of the recombination line emission arising from a hot star wind volume with no specific symmetry, and assumes that the stellar outflow is made of a large number of independent rays. This assumption uses the overwhelming radial nature of line driving in radiatively driven winds, so that the angular correlation length of any two wind points can be assumed to be limited.
We simulate the randomness of the wind structure by allocating randomly to each cone one $1 \mathrm{D}$ output from the radiation hydrodynamics simulations.

Our initial goal was to confirm whether the wind structure generated by the radiative instability can explain the main characteristics of the observed micro-variability of WR and OB stars. We find that under the assumption of spherical symmetry, the synthetic LPV is present only in the profile wings, contrasting with the observations. Relaxing our approach to the full non-symmetric case, implemented with the "patch method" and a cone angle of ca. 3 deg, we obtain a satisfactory agreement between our theoretical profile calculations and the observations, in so far as the migrating nature of profile sub-peaks and profile fluctuations from the mean are concerned.

Being confident about our method and its potential to reveal new conceptual insights into the properties of hot star winds, we wish to go further by investigating a wide range of model parameters, both concerning the wind physics as implemented in the radiation hydrodynamics model ZEUS-2D, but also in the emission line profile treatment, to allow studies for a variety of ionic species probing different heights of the wind. Hence, in future works, we will parameterise the handling of the line emission region in order to select the wind heights contributing to the profile. Also, although we have not included any source of opacity in the present work to keep to the essentials of the line emission processes, we will address this issue in the coming studies. We will first treat the rather trivial case of continuum opacity, and then develop a method for including the line opacity effects, since their influence has been highlighted recently in works dealing with X-ray emission in hot star winds (Ignace \& Gayley 2001; Owocki \& Cohen 2001).

The observational incentive of this and future studies is clearly focused on the case of WR stars, even though the method presented here applies equally well to OB stars. This partly arises from the fact that WR stars reveal exclusively an emission profile micro-variability, while OB stars often show a composite of micro and macro variability, making it hard to disentangle. Note that in principle, micro-variability should also be observable in OB emission lines and our current failure to unveil it may just be due to the limited signal and resolution of our observations.

Finally, our current handling of the radiation force is strictly appropriate for the optically thin winds of $\mathrm{OB}$ stars, so our future work will provide a way of accounting for the multiple nature of line scattering in WR winds and increase the consistency of our approach for the WR objects. We will try especially to provide a satisfactory explanation to the observed slowly migrating variable features seen in all WR wind emission line profiles observed to date. It is commonly believed that this apparently slow acceleration of WR wind structures is a genuine reflection of their dynamical behaviour. We will try to see whether modifications to the radiation hydrodynamics model can provide an extended wind acceleration and test the impact 
on the predicted variability. It becomes clear that although one could argue at first that studying the variability of hot star winds can lead only to refinements of current wind theories, hindsight vividly demonstrates the potential of time dependent studies to constrain the overall wind velocity and acceleration, in a finer way than ever done by steady-state approaches.

Acknowledgements. All hydrodynamics simulations were performed on the basis of the ZEUS-2D code developed at the National Centre for Supercomputing Applications (NCSA) at the University of Urbana, Illinois. Part of this work was carried out while LD held a European Space Agency fellowship at the Université Laval, Québec, Canada. LD acknowledges financial support from the Université Laval for visits to the Bartol Research Institute. SO acknowledges support of NASA grant NAGW5-3530 and NSF grant AST-0097983, awarded to the University of Delaware.

\section{Appendix A: Description of the radiation hydrodynamics code}

\section{A.1. The code itself}

The hydrodynamics code used as the basis of our work is ZEUS-2D, developed by Stone \& Norman (1992). Magnetism and radiation modules were switched off during the calculations so that only the hydrodynamics structure of the code was used. ZEUS-2D is a finite difference, Eulerian code, adaptable to various geometries, although the present work will only consider one-dimensional simulations (in spherical coordinates). The advection scheme used is the piecewise parabolic method introduced by Woodward \& Colella (1984). This is preferred over the Van Leer method as we have found that the latter has a tendency to smooth out the small scale instability structures significantly. No linear nor artificial viscosity is introduced, but the code does use a quadratic viscosity within shock structures generated by the instability. We assume that the wind is also isothermal, so that the sound speed is everywhere equal to its photospheric value. This approximation is appropriate since radiative heating and cooling are very rapid in these dense winds. Moreover, gas pressure plays only a minor role in the dynamics of hot star winds (apart from the photosphere region).

Starting from this basic picture, the radiation force as expressed by CAK was implemented, together with suitable boundary conditions. At the outer boundary, we simply advect the velocity and density by using a constant velocity gradient. At the lower boundary, the photosphere, we extrapolate the velocity inward assuming a constant velocity gradient, and fix the density to a value that is typically about a factor ten above the sonic point density estimated for a steady CAK wind with the assumed linedriving parameters. Gridding is a big issue for the proper modelling of the lower boundary as the wind goes supersonic within a few scale heights of the photosphere. This lower boundary is the anchor for the wind, because there the forces in action are gravity and thermal pressure only, therefore devoid of any radiative instability under all circumstances (there are fatal consequences otherwise).

In the latter part of the work, we use a line opacity distribution (see CAK; Abbott 1980, 1982) which accounts for the opacity cut-off $\kappa_{\max }$ of OCR, but assumes no line overlap (the total contribution from all lines is just a cumulative sum of all individual ones),

$N(\kappa)=\frac{1}{\kappa_{0}}\left(\frac{\kappa}{\kappa_{0}}\right)^{\alpha-2} \mathrm{e}^{-\kappa / \kappa_{\max }}$

Note again that the present work will aim in the longer term to be applicable to WR winds, though presently we restrict our study to optically thin winds where multiple scattering events are out-numbered by single scattering ones. There is at present no formalism that proposes a numerical method for studying the radiative instability in the case of optically thick winds (see Gayley \& Owocki 1995; Gayley et al. 1995 for recent publications on the radiative processes of wind driving in WR stars, or optically thick outflows in general).

We can use the above opacity distribution to recalculate the CAK force, which we now call the Sobolev force:

$g_{\mathrm{Sob}}(r)=\frac{C(r) F(r) N_{0} \Gamma(\alpha)}{(1-\alpha) c}\left(\frac{1}{\rho c} \frac{\mathrm{d} v}{\mathrm{~d} r}\right)^{\alpha}\left(\frac{\left(1+\tau_{\max }\right)^{1-\alpha}-1}{\tau_{\max }^{1-\alpha}}\right)$

where

$\tau_{\max }=\frac{\rho \kappa_{\max } v_{\mathrm{th}}}{\mathrm{d} v / \mathrm{d} r} ; F(r)=\frac{L}{4 \pi r^{2}} ; N_{0}=\left(\frac{\kappa_{0} v_{\mathrm{th}}}{c}\right)^{1-\alpha}$

and $C(r)$ is the finite-disk correction factor (Friend \& Abbott 1986; Pauldrach et al. 1986):

$C(r)=\frac{1-(1-\beta)^{1+\alpha}}{\beta(1+\alpha)} ; \quad \beta=\left(1-\frac{v / r}{\mathrm{~d} v / \mathrm{d} r}\right)\left(\frac{R_{\star}}{r^{2}}\right)$.

Hydrodynamics simulations making use of this radiative force are intrinsically stable, with wind properties that depend on the CAK power index $\alpha$, the opacity normalisation $\kappa_{\mathrm{O}}$, and the cutoff opacity $\kappa_{\max }$. This opacity cutoff sets the maximum opacity accounted for in the force calculation, and therefore puts an upper bound on the maximum force we allow the radiation to impart to matter. It was originally introduced by OCR in pure-absorption simulations to moderate the velocity jump and the associated steep rarefaction above the photosphere resulting from the abrupt increase in the "direct" term of the radiation force. This quantity turns out to be crucial for the wind stability and allowing it to tend to infinity can lead to large and probably unrealistic velocity jumps. So, starting from a sufficiently low value of $\kappa_{\max }$, we compute the wind structure using the above expression for the radiative force. This is then used as an initial condition for calculations made with the Smooth Source Function approximation of Owocki (1991), which provides the following expression for the radiative force (see this paper for the details on the radiative force and the boundary conditions used, as well as OCR and Owocki \& Puls 1996):

$g_{\mathrm{rad}}(r)=\int_{0}^{1} \mathrm{~d} y^{\prime}\left(g_{\mathrm{dir}}\left(r, y^{\prime}\right)+g_{\mathrm{diff}}\left(r, y^{\prime}\right)\right)$ 


$$
\begin{aligned}
& g_{\text {dir }}(r, y)= \frac{N_{0} F(r) \Gamma(\alpha)}{c}\left(\frac{v_{\mathrm{th}}}{c}\right)^{\alpha} \\
& \times\left(\int_{-\infty}^{\infty} \mathrm{d} x p(x, y, r) t_{+}^{-\alpha}(x, y, r)\right) \\
& g_{\mathrm{diff}}(r, y)= \frac{N_{0} F(r) \Gamma(\alpha)}{2\left(1+\mu_{\star}\right) c}\left(\frac{v_{\mathrm{th}}}{c}\right)^{\alpha} \\
& \quad \times\left(\int_{-\infty}^{\infty} \mathrm{d} x p(x, y, r)\left[t_{-}^{-\alpha}(-x, y, r)-t_{+}^{-\alpha}(x, y, r)\right]\right)
\end{aligned}
$$

where the forward and backward frequency-dependent integrated optical depths are:

$$
\begin{aligned}
& t_{+}(x, y, r)=\frac{1}{\kappa_{\max }}+\frac{\phi(x)}{\sigma_{\mathrm{e}}}+\int_{R_{\star}}^{r} \frac{\mathrm{d} r^{\prime}}{\mu_{y}\left(r^{\prime}\right)} \rho\left(r^{\prime}\right) p\left(x, y, r^{\prime}\right) \\
& t_{-}(-x, y, r)=\frac{1}{\kappa_{\max }}+\int_{r}^{\infty} \frac{\mathrm{d} r^{\prime}}{\mu_{y}\left(r^{\prime}\right)} \rho\left(r^{\prime}\right) p\left(x, y, r^{\prime}\right) .
\end{aligned}
$$

We have used for space reduction purposes the profile function $p(x, y, r)=\phi\left(x-\frac{\mu_{y}(r) v(r)}{v_{\mathrm{th}}}\right), \phi(x)$ being chosen as a Gaussian profile, with $\mu_{y}(r)$ the local direction cosine of the ray with impact parameter $y$ at radius $r$, i.e. $\mu_{y}(r)=\sqrt{1-y\left(R_{\star} / r\right)^{2}}$.

As we see, this form of the radiative force allows for two components to contribute, a direct and a diffuse term. The first accounts for the core intensity attenuation alone, and corresponds to the pure absorption case of OCR. It represents most of the radiative force strength at all points except in the subsonic region. The second represents the net force resulting from line scattering in the forward and backward directions within the Sobolev resonance zone. This plays a particularly important role near the wind base as well as within the wind where it leads to enhanced stability in numerical simulations over pure absorption models. In the case of optically thick winds, one needs to allow incoming photons not only from the stellar disk, but rather from all directions. The contribution from the full solid angle is then suited for the treatment of multiple line scattering, in which a photon after being first scattered at a given location, by a given line in a given direction, can be scattered again at another location, in another line and into another direction. For each of these scattering events, a diffuse term could in principle enter if an asymmetry in the inward-outward escape probabilities exists.

Computation of the line force requires a timeconsuming double integration over frequency $x$ and radius $r$ - to avoid a third integral over the direction coordinate $y$, one typically assumes just a single-ray quadrature that intersects the stellar disk with $y=1 / 2$. Due to the profile weighting of the integrand in the expressions of $g_{\text {diff }}(x, y, r)$ and $g_{\text {dir }}(x, y, r)$, we restrict the frequency integration range at each radius to the values of $\mu_{y}(r) v(r) / v_{\text {th }}$ at the left and right side of the corresponding numerical cell. A more accurate treatment integrating from naught to the maximum value of $\mu_{y}(r) v(r) / v_{\text {th }}$ in the wind can increase the computing time by a factor ten, without significantly changing the results.

Also, the interdependence of the forward and backward opacities can be used to shorten the computational work. Indeed, one needs only to calculate $t_{+}(x, y, r)$ from the lower to the upper boundary, to then get the values of $t_{-}(x, y, r)$ from the upper to the lower boundary (this way, we get a reduction of two in the computing time).

With this force included, we let the code run without any additional perturbation for some 50000 s, i.e. until structure has built up over the entire simulated domain (this time varies with the position of the outer boundary). We then have the choice among (1) letting this structured pattern evolve and advect on its own, (2) introducing stochastic fluctuations of the base velocity, or (3) introducing a periodic sound wave perturbation at the photosphere. The last has, in favourable cases, the amazing effect of tuning the wind variability to the period of perturbation.

We now present the model we use in this paper for our line profile simulations assuming a structured wind layout.

\section{A.2. Test model: Time series and snapshots}

In this and subsequent works, our intention is to provide a connection between theoretical predictions and spectroscopic observations of hot star winds. Observations ideally cover a full night, say ten hours. The spatial coverage of the wind described in Sect. 3 consists in a large number of independent radial rays, whose structure is allocated randomly from one-dimensional snapshots calculated by ZEUS-2D. To reduce the possibility of identical structure allocation, we write one output every $100 \mathrm{~s}$, and cover a total time of $80000 \mathrm{~s}$. For the first time profile computation, we choose the data for each ray among the first 400 datasets. We then calculate one hundred profiles, incrementing the index of each ray by 4 at every time step, to be in line with the time gap between successive observations on telescopes.

The nature of the variability we aim at constraining is stochastic. Hence, we make comparisons of each computed profile with the global average. It is therefore crucial that the total dataset shows an overall common behaviour, to make that profile time average meaningful. Note that this is not a numerical fantasy to ease our task, but it is what observations reveal, a global mean profiles which is globally constant in time with small scale variations of typically $5 \%$.

For the present study, we use one set of common parameters for the radiation hydrodynamics simulations, summarised in Table A.1, which are typical of a supergiant $\mathrm{O}$ star. In subsequent studies, we will vary the key parameters of the radiative instability mechanism to modulate the derived structure and assess the impact on the computed emission profile of the opacity cut-off $\kappa_{\max }$, the nature of the photospheric perturbation, and the formation region of the line considered. 

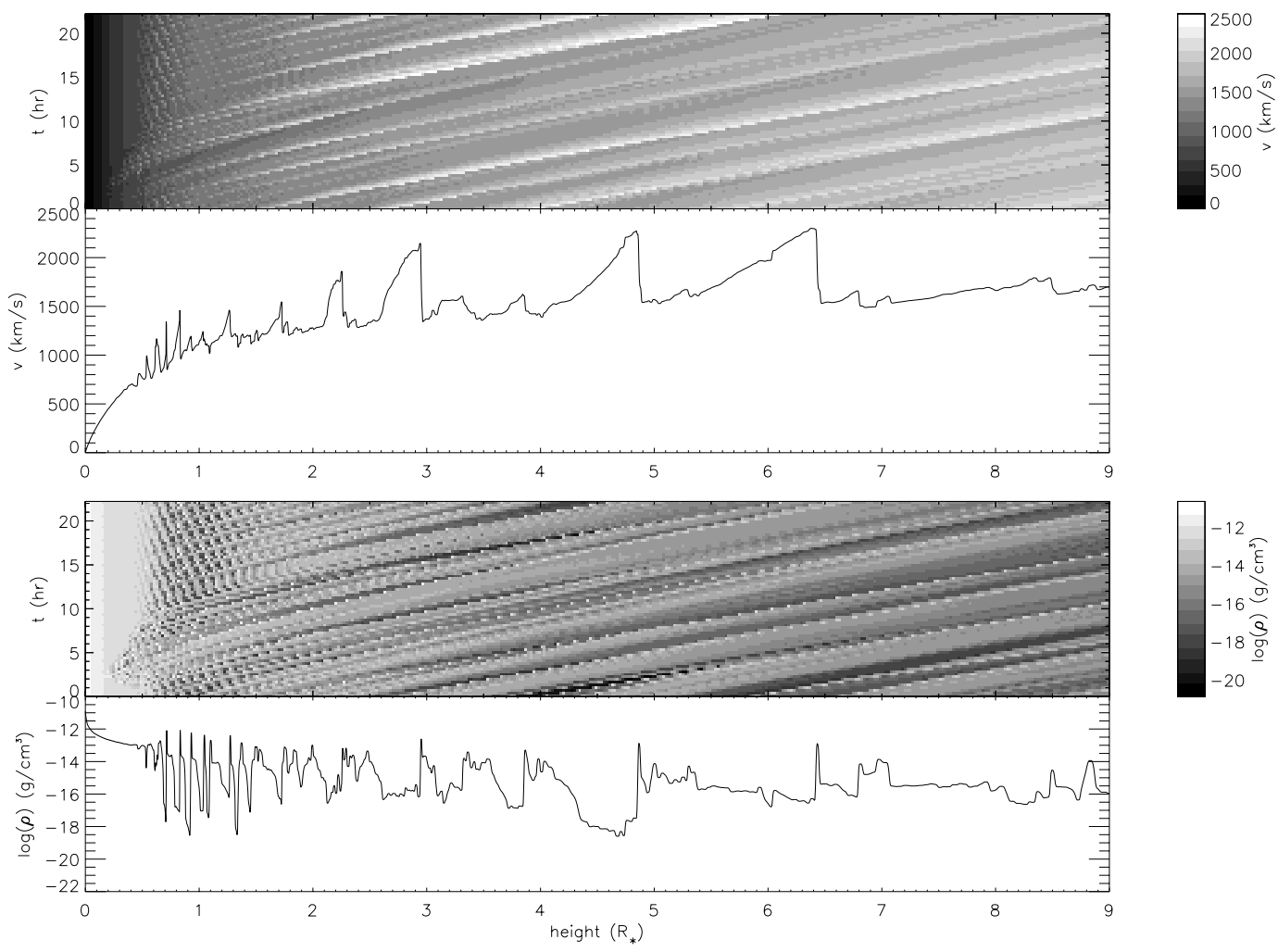

Fig. A.1. Density and velocity layout of the simulated radiatively driven hot star wind. Time series are made up of 800 outputs, taken every $100 \mathrm{~s}$, and covering one period of the sound wave perturbation of the test model. The two cross sections correspond to the last snapshot in the series.

Table A.1. Model parameters input in the radiation hydrodynamics calculations carried out with ZEUS-2D whose wind temporal evolution is displayed in Fig. 1. These stellar parameters are representative of the typical properties of a luminous young massive star with a strong wind. The clumping factor is calculated over the whole wind volume.

\begin{tabular}{lll}
\hline Quantity & Symbol & Value \\
\hline Mass & $M_{\star} / M_{\odot}$ & 42 \\
Luminosity & $L_{\star} / L_{\odot}$ & $10^{6}$ \\
Radius & $R_{\star} / R_{\odot}$ & 19 \\
Photospheric density & $\rho_{\star}\left(\mathrm{g} \mathrm{cm}^{-3}\right)$ & $2.5 \times 10^{-11}$ \\
Sound speed & $a\left(\mathrm{~km} \mathrm{~s}^{-1}\right)$ & 24 \\
Ion thermal velocity & $v_{\mathrm{th}}$ & $0.3 \mathrm{a}$ \\
Opacity constant & $\kappa_{0} v_{\mathrm{th}} / \mathrm{c}\left(\mathrm{cm}^{2} \mathrm{~g}^{-1}\right)$ & 1680 \\
Opacity cut-off & $\kappa_{\mathrm{max}} / \kappa_{0}$ & $1.4 \times 10^{-3}$ \\
Hydrogen mass fraction & $X$ & 0.71 \\
Helium mass fraction & $Y$ & 0.29 \\
CAK power index & $\alpha$ & 0.7 \\
CAK mass loss rate & $\dot{M}_{\mathrm{CAK}}\left(M_{\odot} \mathrm{yr}^{-1}\right)$ & $1.5 \times 10^{-6}$ \\
CAK terminal velocity & $v_{\infty}\left(\mathrm{km} \mathrm{s}^{-1}\right)$ & 2000 \\
Sound wave amplitude & $\delta \rho / \rho$ & 0.01 \\
Sound wave period & $P$ & $5000 \mathrm{~s}$ \\
Clumping factor & $\frac{<\rho^{2}(r, t)>_{r}}{<\rho(r, t)>_{r}^{2}}$ & 12.0 \\
\hline
\end{tabular}

We show in Fig. A.1 the time evolution of the evolved CAK initial condition under a sound wave perturbation at the base, the photosphere, of period $10000 \mathrm{~s}$ and amplitude $1 \%$. To reduce the computing time of such a wind structure calculation, we use a radial spacing that increases with radius, i.e. $\Delta r=0.0007 r$ starting with a spacing $\Delta r=0.001 R_{\star}$. Note that such a gridding can affect in a minor way the properties of the calculated structure in the outer wind. We find a similar wind structure to Owocki (1991) and Feldmeier (1995), i.e. the wind shows a very perturbed structure, consisting of fast and rarefied regions alterning with slow and dense ones. Collisions between these two types of shells occur, dense-dense and rarefied-dense, though not to a large extent. The wind structure is rather quiet compared to highly unstable simulations done with a large value of $\kappa_{\max }$, leading to density contrast no larger than 10000 , with a typical value of 100 in the inner wind regions, and very moderate velocity jumps of at most $500 \mathrm{~km} \mathrm{~s}^{-1}$.

Note that the stabilising effect of the diffuse force on the inner wind regions is clear here, no velocity or density variability being observed shortward of $0.3 R_{\star}$ above the photosphere. This stable region is followed by a very variable and chaotic region which becomes smoother as one moves outwards. This results from the combined effects of the physical merging of shocked regions and the numerical coarseness of the gridding at larger radii.

\section{References}

Abbott, D. C., Bieging, J. H., \& Churchwell, E. 1981, ApJ, 250,645 
Abbott, D. C., Bieging, J. H., Churchwell, E., \& Torres, A. V. 1986, ApJ, 303, 239

Abbott, D. D., \& Lucy, L. B. 1985, ApJ, 288, 679

Brown, J. C., Richardson, L. L., Antokhin, I., et al. 1995, A\&A, 295,725

Castor, J. I., Abbott, D. C., \& Klein, R. I. 1975, ApJ, 195, 157 $(\mathrm{CAK})$

Cranmer, S. R., \& Owocki, S. P. 1996, ApJ, 462, 469

Chlebowski, T. 1989, ApJ, 342, 1091

Dessart, L. 1999, Ph.D. Thesis, Univ. College London

Eversberg, T., Lépine, S., \& Moffat, A. F. J. 1998, ApJ, 494, 799

Feldmeier, A. 1995, A\&A, 299, 523

Feldmeier, A., Puls, J., \& Pauldrach, A. 1997, A\&A, 322, 878

Friend, D., \& Castor, J. 1983, ApJ, 272, 259

Fullerton, A. W., \& Owocki, S. P. 1992, in ed. L. Drissen, C. Leitherer , \& A. Nota, Non-isotropic and variable outflows from stars (ASP San Francisco), 177

Gayley, K. G., \& Owocki, S. P. 1995, ApJ, 446, 801

Gayley, K. G., \& Owocki, S. P. 2000, ApJ, 537, 461

Hillier, D. J., Kudritzki, R. P., Pauldrach, A. W., et al. 1993, A\&A, 176, 117

Hillier, D. J., \& Miller, D. L. 1998, ApJ, 496, 407

Ignace, R., \& Gayley, K. G. 2001, submitted to ApJ

Koesterke, L., \& Hamann, W.-R. 1995, A\&A, 299, 503

Lépine, S., \& Moffat, A. F. J. 1999, ApJ, 514, 909

Lépine, S., Moffat, A. F. J., St-Louis, N., et al. 2000, AJ, 120, 3201
Lucy, L. B., \& Solomon, P. 1970, ApJ, 159, 879

Lucy, L. B. 1982, ApJ, 255, 278

Lucy, L. B. 1984, ApJ, 274, 372

Lucy, L. B., \& White, R. 1980, ApJ, 241, 300

Owocki, S. P., \& Rybicki, G. B. 1984, ApJ, 284, 337

Owocki, S. P., \& Rybicki, G. B. 1985, ApJ, 299, 265

Owocki, S. P., Castor, J. I., \& Rybicki, G. B. 1988, ApJ, 335, 914 (OCR)

Owocki, S. P. 1991, in Stellar Atmospheres: Beyond Classical Models, 235

Owocki, S. P., \& Puls, J. 1996, ApJ, 462, 894

Owocki, S. P., Cranmer, S. R., \& Gayley, K. G. 1996, ApJ, 472, 115

Owocki, S. P., Cranmer, S. R., \& Fullerton, A. W. 1997, ApJ, 453, 37

Owocki, S. P. 1999, in Variable and Non-spherical Stellar Winds in Luminous Hot Stars, 294

Owocki, S. P., \& Cohen, D. H. 2001, ApJ, 559, 1108

Pauldrach, A., Puls, J., \& Kudritzki, R. P. 1986, A\&A, 164, 86

Robert, C. 1992, Ph.D. Thesis, Univ. Montréal

Rybicki, G. B., Owocki, S. P., \& Castor, J. I. 1990, ApJ, 349, 274

Stone, J. M., \& Norman, M. J. 1992, 80, 753

Springmann, U. 1994, A\&A, 289, 505

Woodward, P. R., \& Colella, P. 1984, J. Comput. Phys., 54, 115 